\title{
Effects of Material Symmetry on the Coefficients of Transport in Anisotropic Porous Media
}

\author{
Jacob Bear, Leonid G. Fel and Yoram Zimmels * \\ Department of Civil and Environmental Engineering, \\ Technion - Israel Institute of Technology, Haifa, 32000 Israel
}

\begin{abstract}
The objective of this article is to highlight certain features of a number of coefficients that appear in models of phenomena of transport in anisotropic porous media, especially the coefficient of dispersion, the 2 nd rank tensor $D_{i j}$, and the dispersivity coefficient, the 4 th rank tensor $a_{i j k l}$, that appear in models of solute transport. Although we shall focus on the transport of mass of a dissolved chemical species in a fluid phase that occupies the void space, or part of it, the same discussion is also applicable to transport coefficients that appear in models that describe the advective mass flux of a fluid and the diffusive transport of other extensive quantities, like heat. The case of coupled processes, e.g. the simultaneous transport of heat and mass of a chemical species, are also considered. The entire discussion will be at the macroscopic level, at which a porous medium domain is visualized as homogenized continuum.
\end{abstract}

\section{The coefficient of dispersion, $\mathrm{D}_{i j}$}

We consider the transport by the mechanism of dispersion of a solute in a fluid that occupies the void space of a porous medium, or part of it. The coefficient of dispersion, $\mathrm{D}_{i j}$, appears in the Fickian-type expression for the dispersive flux of a solute (e.g. Bear, 1961)

$$
J_{i}=-\mathrm{D}_{i j} \frac{\partial c}{\partial x_{j}}, \quad i, j \equiv x, y, z, \quad \text { or } \quad i, j \equiv 1,2,3
$$

where $J_{i}$ denotes the $i$ th component of the solute flux vector $\mathbf{J}$ (三 solute mass passing through a unit area of fluid in the porous medium cross-section, per unit time), and $c$ is the solute's concentration (= mass of solute per unit volume of fluid). Furthermore, we use the term flux as an abbreviation for flux density. In (1), and everywhere else in this article, Einstein summation convention is

\footnotetext{
${ }^{*}$ Two months after completing this paper, our colleague Prof. Yoram Zimmels passed away.
} 
applicable, unless the sum symbol is used. The coefficient $D_{i j}$ is a 2 nd rank tensor, relating the vector $\mathbf{J}$ to the vector $\nabla c$.

Equation (11) is valid for the general case of an anisotropic porous medium, with the isotropic medium as a special case. The dispersion coefficient is characterized by:

1. In thermodynamics, the rate of entropy production, $\dot{\mathcal{S}}$, is related to the thermodynamic driving force, $\mathbf{X}$, and the thermodynamic flux, $\mathbf{Y}$, called "conjugated flux and force" (De Groot and Mazur, 1962), by $\dot{\mathcal{S}}=Y_{i} X_{i}$. Here, the flux of the solute, $\mathbf{J}$ is driven by $-\nabla c$, which acts as a "driving force." In this case, the rate of entropy production can be expressed by

$$
\dot{\mathcal{S}}=\chi\left[\left(-\mathrm{D}_{i j} \frac{\partial c}{\partial x_{j}}\right)\right] \times \chi\left[\left(-\frac{\partial c}{\partial x_{i}}\right)\right] \geq 0, \quad \text { or } \quad \mathrm{D}_{i j} \frac{\partial c}{\partial x_{j}} \frac{\partial c}{\partial x_{i}} \geq 0
$$

in which, $\mathbf{Y}=\chi \mathbf{J}$ and $\mathbf{X}=-\chi \nabla c$. The (dimensional) parameter, $\chi$, depends on the considered transport phenomenon. Hence, the $\mathrm{D}_{i j}$-matrix is non-negative definite.

2. The $\mathrm{D}_{i j}$-matrix is symmetric, i.e.,

$$
\mathrm{D}_{i j}=\mathrm{D}_{j i}
$$

The above two statements are consequences of the fact that $\mathbf{X}$ and $\mathbf{J}$ are thermodynamically conjugated force and flux (De Groot and Mazur, 1962), i.e., they satisfy

$$
\frac{\partial J_{i}}{\partial X_{j}}=\frac{\partial J_{j}}{\partial X_{i}}
$$

We may comment here that the above considerations can also be applied to the hydraulic conductivity tensor, $\mathrm{K}_{i j}$, which is a $2 \mathrm{nd}$ rank tensor that appears in Darcy's law, for the general case of an anisotropic porous medium, say,

$$
q_{i}=-\mathrm{K}_{i j} \frac{\partial h}{\partial x_{j}}
$$

where $q_{i}$ denotes the $i$ th component of the flux vector (= discharge per unit area of porous medium per unit time), $h$ denotes the piezometric head, and the vector $-\nabla h$ is the driving force. Here also, the $\mathrm{K}_{i j}$-matrix is positive definite and symmetric, i.e., $\mathrm{K}_{i j}=\mathrm{K}_{j i}$. It is interesting to note that we may have cases with components $\mathrm{K}_{i j} \leq 0$ for $i \neq j$. This is a consequence of the positiveness of the principal minor,

$$
K_{11} K_{22}-K_{12}^{2} \geq 0, \quad \Rightarrow \quad-\sqrt{K_{11} K_{22}} \leq K_{12} \leq \sqrt{K_{11} K_{22}}
$$

For an isotropic porous medium, $\mathrm{K}_{i j}=\mathrm{K} \delta_{i j}$, with $\delta_{i j}$ denoting the Kronecker delta.

The above conclusions are valid also for the coefficient of thermal conductivity, the coefficient of (mass) diffusivity, and the dispersion coefficient of any extensive quantity transported in the fluid that occupies the void space, or part of it. For example: 
1. Mass Transport of a dissolved solute. Such mass can be transported by advection with the moving fluid (not considered here), by dispersion (as described by (1D) and by (molecular) diffusion. For a dilute system, the latter mass flux is described by Fick's law (for a porous medium),

$$
J_{i}^{\gamma}=-\mathcal{D}_{i j}^{\gamma *} \frac{\partial c}{\partial x_{j}},
$$

in which $J_{i}^{\gamma}$ denotes the $i$ th component of mass flux of a dissolved $\gamma$-species (mass of $\gamma$ per unit area of porous medium per unit time), $c$ denotes the concentration of that species, and $\mathcal{D}_{i j}^{\gamma *}$ denotes the coefficient of molecular diffusivity of $\gamma$ in the porous medium. It takes into account the coefficient of molecular diffusion of $\gamma$ in the fluid as a continuum, the porosity of the porous medium and the tortuosity of the void space within the latter (e.g. Bear and Bachmat, 1990, p. 193). The matrix $\mathcal{D}_{i j}^{\gamma *}$ is symmetric and positive definite.

2. Heat transport. Although heat can be transported also by the solid matrix, we focus on the case in which the latter is thermally an insulator. Heat can then be transported by advection with the moving fluid (not discussed here), by thermal dispersion and by thermal conduction (which is a diffusive-type flux), described by (the averaged) Fourier law,

$$
J_{i}^{H}=-\lambda_{i j}^{H *} \frac{\partial T}{\partial x_{j}}
$$

in which $J_{i}^{H}$ denotes the $i$ th component of the heat flux by conduction (heat per unit area of porous medium per unit time), $T$ is the temperature, and $\lambda_{i j}^{H *}$ denotes the $i j$-component of the coefficient of thermal conductivity of the porous medium. Again, the latter takes into account the thermal conductivity of the fluid, as well as the porosity and tortuosity of the void space occupied by the fluid. The matrix $\lambda_{i j}^{H *}$ is symmetric and positive definite.

When the fluid occupying the void space is moving, heat is also transported by advection, with the average velocity of the fluid. and by thermal dispersion. In analogy to (11), the thermal dispersive flux is expressed by

$$
J_{d i s p, i}^{H *}=-\mathrm{D}_{i j}^{H *} \frac{\partial T}{\partial x_{j}}
$$

where $J_{d i s p, i}^{H}$ denotes the $i$ th component of the thermal dispersive flux vector (= heat per unit area of porous medium per unit time), and the vector $-\nabla T$ is the driving force. The $\mathrm{D}_{i j}^{H *}$-matrix is symmetric and positive definite.

Although, we have extended the conclusion about the coefficients being positive definite and symmetric, to a number of transport coefficients, there is a basic difference between the coefficients 
of of hydraulic conductivity, $\mathrm{K}_{i j}$, of mass diffusivity, $\mathcal{D}_{i j}^{\gamma *}$, and of thermal diffusivity in a porous medium, $\lambda_{i j}^{H *}$, as compared to those of (mass) dispersion, $\mathrm{D}_{i j}$, and thermal dispersion, $\mathrm{D}_{i j}^{H}$ :

- $\mathrm{K}_{i j}$ depends on the geometry of the void space through which the water flows (tortuosity and width of pathways), in addition to fluid properties, such as density and dynamic viscosity.

- $\mathcal{D}_{i j}^{\gamma *}$ and $\lambda_{i j}^{H *}$ depend on the geometry of the fluid pathways, as well as the diffusivity and thermal conductivity in the fluid.

In all these cases, the geometry of the fluid-occupied domain is expressed by a scalar that represents the width of the pathways, and a 2nd rank tensor called tortuosity that represents the effect of the tortuous fluid pathways. Bear $(1972$, p. 111) showed that the tortuosity is a 2nd rank symmetric tensor.

- The coefficients of mass and thermal dispersion, $\mathrm{D}_{i j}$ and $\mathrm{D}_{i j}^{H *}$, respectively, are functions not only of the geometry of the void space, but also of the velocity field within the porous medium domain.

We have mentioned several times that a considered fluid may occupy only part of the void space, at some fluid saturation (= volume of fluid per unit volume of void space). Wherever a coefficient depends on the configuration of the void space, it also depends on the saturation of the considered fluid. This aspect is discussed in Sec. 4,

The last two coefficients are discussed in the next section.

\section{The dispersivity, $a_{i j k l}$}

The dispersivity, $a_{i j k l}$, is related to the coefficient of dispersion $\mathrm{D}_{i j}$ by (e.g. Bear, 1972, p. 610)

$$
\mathrm{D}_{i j}=a_{i j k l} \frac{V_{k} V_{l}}{V}
$$

where $V_{k}$ denotes the $k$ th component of the fluid's velocity vector, $\mathbf{V}$, with $V \equiv|\mathbf{V}|$. The coefficient $a_{i j k l}$ is a fourth rank tensor having the following properties:

1. From the expression for the rate of entropy production, $\dot{\mathcal{S}}$, and following the discussion leading to (2), we have

$$
\dot{\mathcal{S}}=Y_{i} X_{i}=\chi\left(-\mathrm{D}_{i j} \frac{\partial c}{\partial x_{i}}\right) \times \chi\left(-\frac{\partial c}{\partial x_{j}}\right)=\chi^{2} a_{i j k l} \frac{\partial c}{\partial x_{i}} \frac{\partial c}{\partial x_{j}} \frac{V_{k} V_{l}}{V} \geq 0
$$

from which it follows that $a_{i j k l}$ is positive definite. Thus, all principal minors of $a_{i j k l}$ are positive. 
2. It has $3^{4}=81$ components in a 3 -dimensional porous medium domain, constrained by $2^{\left(3^{2}\right)}-$ $1=511$ inequalities.

3. It is invariant under the following permutation of indices (see (11)),

$$
a_{i j k l}=a_{i j l k}, \quad a_{i j k l}=a_{j i k l}
$$

Hence, only 36 components are independent of each other. Furthermore, there are $2^{6}-1=63$ constraining inequalities.

Let us apply the above conclusions to an isotropic porous medium domain and to two anisotropic ones.

\subsection{Isotropic porous medium}

For this case, the 36 independent components reduce to two. This can be shown (Sirotine and Chaskolskaya, 1984, p. 651-2) by noting that the fourth rank tensor satisfies the relationships (12) and is invariant under the the action of full rotational (orthogonal) symmetry group $\mathrm{O}(3)$. In the case considered here, the $a_{i j k l}$-tensor can be represented by the matrix $p_{\alpha \beta}$, with

$$
\alpha, \beta=1,2,3 \quad \text { representing } \quad x x, y y, z z, \quad \alpha, \beta=4,5,6 \quad \text { representing } y z, z x, x y,
$$

is:

$$
\left(\begin{array}{cccccc}
p_{11} & p_{12} & p_{12} & 0 & 0 & 0 \\
p_{12} & p_{11} & p_{12} & 0 & 0 & 0 \\
p_{12} & p_{12} & p_{11} & 0 & 0 & 0 \\
0 & 0 & 0 & p_{44} & 0 & 0 \\
0 & 0 & 0 & 0 & p_{44} & 0 \\
0 & 0 & 0 & 0 & 0 & p_{44}
\end{array}\right), \quad p_{44}=\frac{p_{11}-p_{12}}{2} .
$$

Denoting $p_{44}=\left(a_{L}-a_{T}\right) / 2, p_{11}=a_{L}$, and $p_{12}=a_{T}$, we obtain:

$$
a_{i j k l}=a_{T} \delta_{i j} \delta_{k l}+\frac{a_{L}-a_{T}}{2}\left(\delta_{i k} \delta_{j l}+\delta_{i l} \delta_{j k}\right)
$$

and

$$
\mathrm{D}_{i m}=a_{T} V \delta_{i m}+\left(a_{L}-a_{T}\right) \frac{V_{i} V_{m}}{V} .
$$

where the two independent coefficients are the longitudinal dispersivity, $a_{L}$, and the transversal dispersivity, $a_{T}$ (e.g. Bear, 1972, p. 611). Furthermore, from the positive definiteness of $a_{i j k l}$, it follows

$$
a_{L} \geq 0, \quad a_{T} \geq 0
$$




\subsection{Axially symmetric porous medium}

This case, also called axially symmetric anisotropy, occurs, for example, when the porous medium is made of many, relatively thin isotropic porous layers. The same kind of material is obtained when we fill space by parallelepiped solid bodies, say boxes, $a \times a \times b$, in the $x, y, z$, directions, respectively, with equal spacing between all boxes. The $z$-axis is an axis of symmetry. In this case, the 36 independent components of $a_{i j k l}$ reduce to six (Sirotine and Chaskolskaya, 1984, p. 652),

$$
\left(\begin{array}{cccccc}
p_{11} & p_{12} & p_{13} & 0 & 0 & 0 \\
p_{12} & p_{11} & p_{13} & 0 & 0 & 0 \\
p_{31} & p_{31} & p_{33} & 0 & 0 & 0 \\
0 & 0 & 0 & p_{44} & 0 & 0 \\
0 & 0 & 0 & 0 & p_{44} & 0 \\
0 & 0 & 0 & 0 & 0 & p_{66}
\end{array}\right), \quad p_{66}=\frac{p_{11}-p_{12}}{2}
$$

where we have made use of (13), taking the $z$-axis as the axis of symmetry. The number 6 is reached by considering fourth rank tensors that satisfy the relationships (12) and are invariant under the the action of the uniaxial symmetry group, $D_{\infty h}$.

Furthermore, because of the positiveness of the minors of the above matrix, the 6 constraints satisfy:

$$
p_{11}, p_{33}, p_{44} \geq 0, \quad p_{11} \geq p_{12}, \quad p_{11} p_{33} \geq p_{13} p_{31}, \quad \operatorname{det}\left(\begin{array}{ccc}
p_{11} & p_{12} & p_{13} \\
p_{12} & p_{11} & p_{13} \\
p_{31} & p_{31} & p_{33}
\end{array}\right) \geq 0
$$

where we have made use of (13).

Another form of presenting $a_{i j k l}$, with the vector e (components $e_{i}$ ) denoting the direction of the axis of symmetry, is

$$
\begin{aligned}
a_{i j k l}= & a_{1} \delta_{i j} \delta_{k l}+\frac{a_{2}}{2}\left\{\delta_{i k} \delta_{j l}+\delta_{i l} \delta_{j k}\right\}+a_{3} e_{i} e_{j} \delta_{k l}+a_{4} e_{k} e_{l} \delta_{i j}+ \\
& \frac{a_{5}}{2}\left\{e_{i} e_{k} \delta_{j l}+e_{j} e_{k} \delta_{i l}+e_{i} e_{l} \delta_{j k}+e_{j} e_{l} \delta_{i k}\right\}+a_{6} e_{i} e_{j} e_{k} e_{l} .
\end{aligned}
$$

Poreh (1965), using Robertson (1940), derived an expression for $a_{i j k l}$ which missed 3 of the terms appearing in (201). The corresponding $\mathrm{D}_{i j}$ is

$$
\begin{aligned}
D_{i j} V= & a_{1} \delta_{i j}\left(V_{k} V_{k}\right)+a_{2} V_{i} V_{j}+a_{3} e_{i} e_{j}\left(V_{k} V_{k}\right)+a_{4}\left(V_{k} e_{k}\right)\left(V_{l} e_{l}\right) \delta_{i j}+ \\
& \frac{a_{5}}{2}\left\{e_{i}\left(V_{k} e_{k}\right) V_{j}+e_{j}\left(V_{k} e_{k}\right) V_{i}+e_{i}\left(V_{l} e_{l}\right) V_{j}+e_{j}\left(V_{l} e_{l}\right) V_{i}\right\}+a_{6} e_{i} e_{j}\left(V_{k} e_{k}\right)\left(V_{l} e_{l}\right) \\
= & e_{i} e_{j}\left\{a_{6}\left(V_{k} e_{k}\right)^{2}+a_{3} V^{2}\right\}+\delta_{i j}\left\{a_{4}\left(V_{k} e_{k}\right)^{2}+a_{1} V^{2}\right\}+a_{2} V_{i} V_{j}+ \\
& +a_{5}\left(V_{k} e_{k}\right)\left\{e_{i} V_{j}+e_{j} V_{i}\right\} .
\end{aligned}
$$


As an example, consider the case of a layered porous medium domain. This is an axially symmetric case, with the $z$-axis as axis of symmetry. Consider now uniform flow normal to the layers. This means: $e_{1}=e_{2}=0, e_{3}=1$ and $V_{1}=V_{2}=0, V_{3}=V$. For this case, (21) can be written as:

$$
\mathrm{D}_{i j}=\left(\begin{array}{ccc}
\mathrm{a}_{T H V} & 0 & 0 \\
0 & \mathrm{a}_{T H V} & 0 \\
0 & 0 & \mathrm{a}_{L V V}
\end{array}\right) V, \quad \begin{aligned}
& \mathrm{a}_{T H V}=a_{1}+a_{4}, \\
& \mathrm{a}_{L V V}=a_{1}+a_{2}+a_{3}+a_{4}+2 a_{5}+a_{6}
\end{aligned}
$$

For uniform flow parallel to the layers, say, in the $+x$-axis, $e_{1}=e_{2}=0, e_{3}=1$ and $V_{1}=V, V_{2}=$ $V_{3}=0,(21)$ can be rewritten as:

$$
\mathrm{D}_{i j}=\left(\begin{array}{ccc}
\mathrm{a}_{L H H} & 0 & 0 \\
0 & \mathrm{a}_{T H H} & 0 \\
0 & 0 & \mathrm{a}_{T V H}
\end{array}\right) V, \quad \begin{aligned}
& \mathrm{a}_{L H H}=a_{1}+a_{2} \\
& \mathrm{a}_{T H H}=a_{1} \\
& \mathrm{a}_{T V H}=a_{1}+a_{3} .
\end{aligned}
$$

Thus, to fully describe dispersion in a layered porous medium, when the flow is uniform and normal to the layers, we need one longitudinal and one transversal dispersivities. For uniform flow in the layers, we need (another) one longitudinal and two transversal dispersivities. In addition to these 5 coefficients, we need information on the direction of the axis of symmetry, i.e., we need six independent coefficients to completely describe the dispersion in such a domain, when the flows are uniform, wither along the axis of symmetry or normal to it. In the case of general flow through such domain, as shown earlier, we need six independent coefficients, plus information about the axis of symmetry. Note that because of the axial symmetry, only one angle is required to completely define the axis of symmetry.

It is interesting to note that Batchelor (1946), in his work on axisymmetric turbulence, using a method based on invariance, introduced by Robertson (1940), derived a general expression for $\mathrm{D}_{i j}$ that is based on five tensorial terms, $e_{i} e_{j}, \delta_{i j}, V_{i} V_{j}, V_{i} e_{j}, V_{j} e_{i}$, and four scalar functions, $C_{i}$ :

$$
\mathrm{D}_{i j} V=C_{1}(\mathbf{V}, \mathbf{e}) e_{i} e_{j}+C_{2}(\mathbf{V}, \mathbf{e}) \delta_{i j}+C_{3}(\mathbf{V}, \mathbf{e}) V_{i} V_{j}+C_{4}(\mathbf{V}, \mathbf{e})\left\{V_{i} e_{j}+V_{j} e_{i}\right\}
$$

To construct the generic forms of the four scalar function $C_{i}$, where $C_{i}=C_{i}(\mathbf{V}, \mathbf{e}), i=1, . .4$, such that they survive under the action of axisymmetric group, noting that there are only two quadratic-in- $V$ scalar invariants: $V^{2}$ and $\left(V_{k} e_{k}\right)^{2}$, we have:

$$
C_{1}=a_{6}\left(V_{k} e_{k}\right)^{2}+a_{3} V^{2}, \quad C_{2}=a_{4}\left(V_{k} e_{k}\right)^{2}+a_{1} V^{2}, \quad C_{3}=a_{2}, \quad C_{4}=a_{5}\left(V_{k} e_{k}\right)
$$

We have chosen the use of the same notation as in (21) in order to emphasize the explicit equivalence between the two expressions for $\mathrm{D}_{i j}$. Poreh (1965) followed the same analysis as that presented by 
Batchelor (1946). Altogether, the above comments explain why in the literature one can find the numbers four, five and six proposed by different authors. As we have shown, there is no contradiction; $\mathrm{D}_{i j}$ is expressed by five tensorial terms, associated with four quadratic-in- $V$ functions. The latter are constructed by six independent coefficients.

Bear and Bachmat (1990, p. 215) also used Robertson's (1940) method and reached the results of 2 independent coefficients for the isotropic case, and 6 for the axially symmetric one. However, they, erroneously, also assumed symmetry permutations of the couples of indices $i, k$ and $j, l$, which led to only 5 independent scalar coefficients for the latter case. The reason for the additional assumption of symmetry followed from the assumption of analogy between dispersion and elasticity.

\subsection{Analogy with elasticity}

It is often stated that the relationship (10) is analogous to the relationship between the stress $\left(\sigma_{i j}\right)$ and the strain $\left(\varepsilon_{i j}\right)$ for an elastic solid:

$$
\varepsilon_{i j}=C_{i j k l} \sigma_{k l}
$$

in which $C_{i j k l}$ denotes components of the solid's elastic modulus, which is a 4th rank tensor. Here, following the discussion leading to (2), the rate of entropy production, is expressed by:

$$
\dot{\mathcal{S}} \equiv\left(\chi \sigma_{i j}\right)\left(\chi \varepsilon_{i j}\right)=\chi^{2} \sigma_{k l}\left(C_{i j k l} \sigma_{i j}\right)
$$

which leads to the symmetry $C_{i j k l}=C_{k l i j}$, in addition to the validity of (12). Thus, for an isotropic solid, have

$$
C_{i j k l}=A_{1}\left(\delta_{i k} \delta_{j l}+\delta_{i l} \delta_{j k}\right)+A_{2} \delta_{i j} \delta_{k l},
$$

in which $A_{1}$ and $A_{2}$ are scalar coefficients, which are related to the Lamé constants for the elastic solid. Thus, for an isotropic solid, because these two coefficients are properties of the solid material only, they can be expressed in terms of the two coefficients: Young's modulus of elasticity and Poisson's ratio.

In the axially symmetric case (symmetry group $\left.D_{\infty h}\right)$, the total number $(=21)$ of independent components, $C_{i j k l}$ is reduced to 5 . However, in the case of the dispersivity tensor, $a_{i j k l}$, we have shown that the number of independent coefficients is 6 and not 5 . Thus, the assumption of analogy is erroneous.

\subsection{Anisotropic porous medium with tetragonal symmetry}

We consider the case of an anisotropic porous medium with tetragonal symmetry. This kind of symmetry is referred to as $\mathrm{D}_{4 h}$ (Sirotine and Chaskolskaya, 1984, p. 651). As an example of such 
porous medium material, we may consider one that is made up of orderly packed solid boxes $a \times a \times c$ with equal spacing between the boxes in all directions (or cubes with 3 different spaces). For this case, the dispersivity tensor can be described by the matrix representation

$$
\left(\begin{array}{cccccc}
p_{11} & p_{12} & p_{13} & 0 & 0 & 0 \\
p_{12} & p_{11} & p_{13} & 0 & 0 & 0 \\
p_{31} & p_{31} & p_{33} & 0 & 0 & 0 \\
0 & 0 & 0 & p_{44} & 0 & 0 \\
0 & 0 & 0 & 0 & p_{55} & 0 \\
0 & 0 & 0 & 0 & 0 & p_{55}
\end{array}\right),
$$

where we have made use of (13).

In this case, the 36 independent components can be expressed by 7 independent parameters. Furthermore, because of the positiveness of the minors of the above matrix, we have the 10 constraints:

$$
p_{11}, p_{33}, p_{44}, p_{55} \geq 0, \quad p_{11}^{2} \geq p_{12}^{2}, \quad p_{11} p_{33} \geq p_{13} p_{31}, \quad \operatorname{det}\left(\begin{array}{ccc}
p_{11} & p_{12} & p_{13} \\
p_{12} & p_{11} & p_{13} \\
p_{31} & p_{31} & p_{33}
\end{array}\right) \geq 0 .
$$

This is an obvious extension of (39), which presents the case of flow parallel to the layers in an axially-symmetrical case. Altogether, we need information on the directions in which the boxes, $a \times a \times c$, are positioned in space (and this requires information on 2 angles), and then 3 coefficients: (one longitudinal dispersivity coefficient and two different transversal ones) for uniform flow in the $a$ direction, and 2 coefficients (one (different) longitudinal dispersivity and one (different) transversal dispersivity) for uniform flow in the $c$-direction Altogether, 7 independent coefficients (or 5 , if the spatial directions are known).

Another (non-matrix) form of the dispersion tensor, $\mathrm{D}_{i j}$, can be obtained by considering the invariants that can be constructed of the four vectors: the velocity, $\mathbf{V}$ and the three mutually orthogonal unit vector, $\boldsymbol{\alpha}, \boldsymbol{\beta}, \boldsymbol{\gamma}$, such that

$$
\begin{gathered}
\alpha_{k} \beta_{k}=\beta_{k} \gamma_{k}=\gamma_{k} \alpha_{k}=0, \quad \alpha_{k} \alpha_{k}=\beta_{k} \beta_{k}=\gamma_{k} \gamma_{k}=1 \\
\boldsymbol{\gamma}=\boldsymbol{\alpha} \times \boldsymbol{\beta}, \quad \boldsymbol{\beta}=\boldsymbol{\gamma} \times \boldsymbol{\alpha}, \quad \boldsymbol{\alpha}=\boldsymbol{\beta} \times \boldsymbol{\gamma}
\end{gathered}
$$

We also require that the symmetric tensor $\mathrm{D}_{i j}\left(=\mathrm{D}_{j i}\right)$ survives under the action of tetragonal group,

$$
\mathrm{D}_{i j}(\boldsymbol{\alpha})=\mathrm{D}_{i j}(-\boldsymbol{\alpha}), \quad \mathrm{D}_{i j}(\boldsymbol{\beta})=\mathrm{D}_{i j}(-\boldsymbol{\beta}), \quad \mathrm{D}_{i j}(\boldsymbol{\gamma})=\mathrm{D}_{i j}(-\boldsymbol{\gamma}), \quad \mathrm{D}_{i j}(\boldsymbol{\alpha}, \boldsymbol{\beta}, \gamma)=\mathrm{D}_{i j}(\boldsymbol{\beta}, \alpha, \gamma)
$$


With the above in mind, we start by listing all symmetric 2 nd rank tensors built of the four vectors: $\mathbf{V}$ and $\boldsymbol{\alpha}, \boldsymbol{\beta}, \boldsymbol{\gamma}$ :

$$
\begin{gathered}
\delta_{i j}, \quad \alpha_{i} \alpha_{j}, \quad \beta_{i} \beta_{j}, \quad \gamma_{i} \gamma_{j}, \quad V_{i} V_{j}, \\
\alpha_{i} V_{j}+\alpha_{j} V_{i}, \quad \beta_{i} V_{j}+\beta_{j} V_{i}, \quad \gamma_{i} V_{j}+\gamma_{j} V_{i} . \\
\alpha_{i} \beta_{j}+\alpha_{j} \beta_{i}, \quad \beta_{i} \gamma_{j}+\beta_{j} \gamma_{i}, \quad \gamma_{i} \alpha_{j}+\gamma_{j} \alpha_{i} .
\end{gathered}
$$

Actually, because of the relationship (30), only two of these vectors suffice to completely define $\mathrm{D}_{i j}$, provided, these vectors are chosen consistently. Here, once we select $\boldsymbol{\alpha}, \boldsymbol{\beta}$, the vector $\boldsymbol{\gamma}$ is well defined. Accordingly, we obtain

$$
\begin{gathered}
\delta_{i j}, \quad \alpha_{i} \alpha_{j}, \quad \beta_{i} \beta_{j}, \quad V_{i} V_{j} \\
\alpha_{i} V_{j}+\alpha_{j} V_{i}, \quad \beta_{i} V_{j}+\beta_{j} V_{i}, \quad \alpha_{i} \beta_{j}+\alpha_{j} \beta_{i} .
\end{gathered}
$$

Next we have to select coefficients such that when multiplying each of the above terms, contributes a quadratic in $V$ term to the expression of $\mathrm{D}_{i j}$. Furthermore, the resulting $\mathrm{D}_{i j}$ has to satisfy (31). With this last requirement, the terms in (34) have no contribution. The first three terms in (35), require coefficients of the form

$$
A_{1}\left(\left(V_{k} \alpha_{k}\right)^{2}+\left(V_{k} \beta_{k}\right)^{2}\right)+A_{2} V^{2}
$$

in which the $A_{i}$ 's take on different values for each of the first three terms in (35). For the last term in (35), the coefficient is a constant. As for the last term in (36), it can be shown that only one scalar invariant can serve as a coefficient, as otherwise, condition (31) will be violated. Thus, we obtain:

$$
B\left(\left(\alpha_{k} V_{k}\right)\left(\alpha_{i} V_{j}+\alpha_{j} V_{i}\right)+\left(\beta_{k} V_{k}\right)\left(\beta_{i} V_{j}+\beta_{j} V_{i}\right)\right) .
$$

As to the last term in (36), its contribution is:

$$
C\left(\alpha_{k} V_{k}\right)\left(\beta_{k} V_{k}\right)\left(\alpha_{i} \beta_{j}+\alpha_{j} \beta_{i}\right) .
$$

Altogether, we arrive at the final expression, in terms of 7 coefficients:

$$
\begin{aligned}
V D_{i j}= & \left.\left(A_{1}\left(\left(\alpha_{k} V_{k}\right)^{2}+\left(\beta_{k} V_{k}\right)^{2}\right)\right)+A_{2} V^{2}\right) \delta_{i j} \\
& \left.+\left(A_{3}\left(\left(\alpha_{k} V_{k}\right)^{2}+\left(\beta_{k} V_{k}\right)^{2}\right)\right)+A_{4} V^{2}\right)\left(\alpha_{i} \alpha_{j}+\beta_{i} \beta_{j}\right) \\
& +A_{5}\left(\left(\alpha_{k} V_{k}\right)\left(\alpha_{i} V_{j}+\alpha_{j} V_{i}\right)+\left(\beta_{k} V_{k}\right)\left(\beta_{i} V_{j}+\beta_{j} V_{i}\right)\right) \\
& +A_{6}\left(\alpha_{k} V_{k}\right)\left(\beta_{k} V_{k}\right)\left(\alpha_{i} \beta_{j}+\alpha_{j} \beta_{i}\right)+A_{7} V_{i} V_{j} .
\end{aligned}
$$


As an example, consider the case of a porous medium made of boxes, $a \times a \times c$, with $a$ in the $x$ and $y$ directions and $c$ in the $z$-direction, and $\boldsymbol{\alpha}=(1,0,0), \boldsymbol{\beta}=(0,1,0), \boldsymbol{\gamma}=(0,0,1)$. For uniform flow in the $x$ or $y$ directions, i.e., $V_{1}=V, V_{2}=V_{3}=0$, (37) can be written as:

$$
D_{i j}=\left(\begin{array}{ccc}
a_{L H H} & 0 & 0 \\
0 & a_{T H H} & 0 \\
0 & 0 & a_{T V H}
\end{array}\right) V, \quad \begin{aligned}
& a_{L H H}=A_{1}+A_{2}+A_{3}+A_{4}+2 A_{5}+A_{7}, \\
& a_{T H H}=A_{1}+A_{2}+A_{3}+A_{4}, \\
& a_{T V H}=A_{1}+A_{2} .
\end{aligned}
$$

For uniform flow in the $+z$-axis, $V_{1}=V_{2}=0, V_{3}=V$, (37) can be rewritten as:

$$
D_{i j}=\left(\begin{array}{ccc}
a_{T H V} & 0 & 0 \\
0 & a_{T H V} & 0 \\
0 & 0 & a_{L V V}
\end{array}\right) V, \quad \begin{array}{ll}
a_{T H V}= & A_{2}+A_{4} \\
a_{L V V}= & A_{2}+A_{7} .
\end{array}
$$

Thus, to fully describe dispersion in a layered porous medium, when uniform flow takes place in the $+x$ or $+y$ directions, we need one longitudinal and two transversal dispersivities-in the horizontal direction and in the vertical one. For uniform flow in the vertical $(+z$-)direction, we need (another) one longitudinal and one transversal dispersivities. Altogether we need 5 coefficients, plus information - two angles - on the directions of the axes, i.e., we need seven independent coefficients to completely describe the dispersion (i.e., $\mathrm{D}_{i j}$ ) in such a porous medium.

Needless to add that in all cases, the actual values of the various coefficients have to be determined experimentally. The knowledge about the number of independent coefficients in each case facilitates the design of the experiments.

The analysis presented above for the cases of isotropic and anisotropic porous media, can be extended to any other types of anisotropy.

\section{Coupled processes}

\subsection{Conjugate fluxes and forces}

The generalized Newton's law that describes molecular flux of linear momentum, Fourier's law, that describes conductive heat flux, and Fick's law that describes diffusive mass flux of a solute $(=$ molecular diffusion), are particular cases of the general linear law for phenomena of transport in a fluid phase (e.g. Bear and Bachmat, 1990, p. 99)

$$
J_{i}^{n}=-\sum_{j=1}^{3} L_{i j}^{n n} \frac{\partial \Phi^{n}}{\partial x_{j}}, \quad i, j=1,2,3, \quad(\text { or } \quad=x, y, z),
$$

where $J_{i}^{n}$ denotes the $i$ th component of the flux of an extensive quantity $E^{n}$ of a phase, $\Phi^{n}$ is a state variable associated with $E^{n}$ and $L_{i j}^{n n}$ is a coefficient of proportionality. 
The three linear diffusive flux laws mentioned above, state that a nonuniform distribution of the state variable, $\Phi^{n}$ (e.g., temperature), produces a flux of only the corresponding extensive quantity (e.g., heat). However, experimental evidence suggests that, in principle, gradients of state variables, $\Phi^{r}, r \neq n$, corresponding to other extensive quantities, may also contribute to the flux of $E^{n}$. Such phenomena are referred to as coupled phenomena. An example of such phenomenon is the Soret (or thermodiffusion) effect, in which mass flux of a solute in a liquid phase is produced by a temperature gradient, in addition to the flux produced by the gradient of the solute's concentration according to Fick's law. Another example is the Dufour effect, in which heat flux is caused by a concentration gradient, in addition to the heat flux caused by temperature gradient, according to Fourier's law. Actually, the diffusive flux of a component, as expressed by Fick's law, manifests interdependence between the concentrations of components in a multicomponent system,

$$
J_{i}^{\beta}=-\sum_{\alpha=1}^{N} \sum_{\mathrm{J}=1}^{3} \mathcal{D}_{i j}^{\alpha \beta} \frac{\partial c^{\beta}}{\partial x_{j}}, \quad \alpha,=1,2, \ldots, N .
$$

where $N$ denotes the number of dissolved chemical species, and $c^{\beta}$ denotes the concentration of the $\beta$-species. Using the terminology introduced above, we have to regard the mass of a dissolved $\beta$-species as one of the $E^{n}$ extensive quantities.

All the above diffusive flux expressions are valid within the fluid that occupies the void space of a porous medium, or part of it, i.e., at the microscopic level of description. By appropriate averaging, we obtain the corresponding flux laws at the macroscopic, or averaged level. In fact, they have the same linear form as (40), except that $J_{i}^{n}$ denotes the $i$ th component of the flux of an extensive quantity $E^{n}$ of a phase, per unit area of porous medium, $\Phi^{n}$ is the macroscopic state variable associated with $E^{n}$, and the coefficient $L_{i j}^{n n}$ takes into account the porosity and the tortuosity of the porous medium. For short, we shall leave the same symbols also for the macroscopic interpretation, as our discussion focuses on forces, fluxes, and coefficients at the macroscopic level.

We consider $\ell$ extensive quantities, and $\ell$ corresponding fluxes produced by the thermodynamic forces,

$$
X_{j}^{r}=-\frac{\partial \Phi^{r}}{\partial x_{j}}, \quad r=1,2, \ldots, \ell
$$

Each diffusive flux $Y_{i}^{r}$ and the corresponding force $X_{i}^{r}$ are chosen such that they are thermodynamically conjugate force and flux (Sec. 1). For the set of coupled fluxes, we have:

$$
Y_{i}^{q}=\sum_{r=1}^{\ell} \sum_{j=1}^{3} L_{i j}^{q r} X_{j}^{r}, \quad i=1,2,3 ; \quad q=1,2, \ldots, \ell .
$$

From the phenomenological equations (43), it follows that the flux of an extensive quantity, $E^{q}$, is a single-valued function of all the (coupled) thermodynamic forces associated with $E^{q}$. They 
express linear relationships between fluxes and thermodynamic forces.

Equation (43) can be extended to coupled phenomena of a higher order, i.e., when the transport is described by coefficients that are tensors of 3rd and higher ranks. As examples, we may mention the coupling between piezoelectric and piezomagnetic phenomena discussed by Fel (2002).

Before continuing with the discussion of coupled processes, let us make the following important comment. Although the hydraulic conductivity $K_{i j}$, appearing in Darcy's law, and all the coefficients $L_{i j}^{q r}$ appearing in the equation that describes coupled diffusive fluxes, are 2nd rank tensor coefficients, there is a basic difference between them. Although Darcy (1856) obtained his law as an empirical one, it is actually a simplified version of the averaged momentum balance equation of a fluid. This law exists only at the macroscopic (porous medium) level, describing advective flux in a porous medium domain, while all other diffusive flux laws exist in a fluid continuum, i.e. at the microscopic level. When averaged, they become macroscopic diffusive flux laws (of heat, mass of a solute, etc.) in a porous medium domain. Coupling as discussed above exists only among diffusive-type fluxes.

In 1851, Stokes postulated that in (40), the coefficients $L_{i j}^{q q}$ are symmetric with respect to the permutation of the coordinates $i$ and $j$, i.e.

$$
L_{i j}^{q q}=L_{j i}^{q q}
$$

This postulate states that the transformation of a unit force along one axis into a conjugate flux along another axis remains unaltered when those axes are interchanged. In Sec. 1, we have discussed the three coefficients $\mathrm{D}_{i j}, \mathrm{~K}_{i j}$ and $\mathrm{D}_{i j}^{H *}$.

Bear and Bachmat (1990, p. 226) have extended the concept of coupled processes to the macroscopic level in a porous medium domain. They suggested that the concept of coupling, remains valid also at the macroscopic level, provided the coefficients involved, $L_{i j}^{q r}$ corresponding to a phase continuum, be multiplied by the tortuosity of the porous medium.

Let us focus on the cross coefficients for $q \neq r$, which give the flux of $E^{q}$ caused by the force, $\mathbf{X}^{r}$, associated with the gradient of $e^{r}$ (三 the density of $E^{r}$ ). Employing the principle of microscopic reversibility of processes, and methods of statistical mechanics, Onsager (1931) showed that for the linear equations (43), and provided a proper choice is made for the fluxes, $\mathbf{Y}^{q}$, and forces, $\mathbf{X}^{r}$, the phenomenological coefficients are also symmetric in $r$ and $q$, i.e.

$$
L_{i j}^{q r}=L_{j i}^{r q}, \quad q \neq r .
$$

This follows from the symmetry of the composite matrix. For the example, the composite matrix for $\ell=2$ takes the form: 


$$
\left(\begin{array}{ll}
\left(L_{i j}^{11}\right) & \left(L_{i j}^{12}\right) \\
\left(L_{i j}^{21}\right) & \left(L_{i j}^{22}\right)
\end{array}\right) .
$$

These, relationships are known as Onsager's, or Onsager-Casimir's, reciprocal relations. They express a relationship between any pair of cross-phenomena (e.g., thermal diffusion and Dufour effect) arising from simultaneously occurring irreversible processes (e.g., heat conduction and molecular diffusion). According to Onsager, the reciprocal relations, (44), hold under two conditions (e.g., Bear and Bachmat, 1990, p. 103):

1. The relationship between each individual flux and its conjugate thermodynamic force is linear.

2. The fluxes, $\mathbf{Y}^{q}$, and their conjugate forces, $\mathbf{X}^{q}$, should be selected such that

$$
\dot{\mathcal{S}}=\sum_{q=1}^{\ell} \sum_{i=1}^{3} Y_{i}^{q} X_{i}^{q},
$$

where $\dot{\mathcal{S}}$ is the rate of entropy production of the system (De Groot, 1963, Ch. 1).

Equation (47) implies that each $\mathbf{Y}^{q}$ and its conjugate force, $\mathbf{X}^{q}$, must be of the same tensorial rank (not necessarily a vector, as indicated by the single subscript).

By the 2nd law of thermodynamics

$$
\dot{\mathcal{S}} \geq 0 \rightarrow \sum_{(q, r, i, j)} L_{i j}^{q r} X_{j}^{r} X_{i}^{q} \geq 0
$$

Hence, a necessary condition for the validity of (48) is that all diagonal matrices $L_{i j}^{q q}$ are positive definite. Let us consider three special cases of porous media.

\section{$3.2 \quad$ Isotropic porous medium, $O(3)$}

For an isotropic medium, using the example of $i, j,=1,2,3$ and $\ell=2$, e.g., coupled fluxes of heat and solute, we can write the coefficients $L_{i j}^{q r}$ in the matrix form,

$$
\left(\begin{array}{l}
\left(\begin{array}{lll}
a & 0 & 0 \\
0 & a & 0 \\
0 & 0 & a
\end{array}\right)\left(\begin{array}{lll}
c & 0 & 0 \\
0 & c & 0 \\
0 & 0 & c
\end{array}\right) \\
\left(\begin{array}{lll}
c & 0 & 0 \\
0 & c & 0 \\
0 & 0 & c
\end{array}\right) \\
\left(\begin{array}{lll}
d & 0 & 0 \\
0 & d & 0 \\
0 & 0 & d
\end{array}\right)
\end{array}\right)
$$


with $a, c, d$ denoting coefficients, e.g., $a \equiv L_{11}^{11}$, and $d \equiv L_{11}^{22}$. We have here 3 independent coefficients, with the inequalities $a, d \geq 0$, and $a d>c^{2}$.

\subsection{Anisotropy - with uniaxial symmetry, $D_{\infty h}$}

For this case, again, with $i, j,=1,2,3$ and $\ell=2$, the matrix of coefficients, can be represented as

$$
\left(\begin{array}{l}
\left(\begin{array}{lll}
a & 0 & 0 \\
0 & a & 0 \\
0 & 0 & d
\end{array}\right)\left(\begin{array}{lll}
b & 0 & 0 \\
0 & b & 0 \\
0 & 0 & c
\end{array}\right) \\
\left(\begin{array}{lll}
b & 0 & 0 \\
0 & b & 0 \\
0 & 0 & c
\end{array}\right) \\
\left(\begin{array}{lll}
e & 0 & 0 \\
0 & e & 0 \\
0 & 0 & f
\end{array}\right)
\end{array}\right)
$$

Altogether, we have here 6 independent coefficients, which are governed by 6 inequalities:

$$
a, d, e, f \geq 0, \quad a e \geq b^{2}, \quad d f \geq c^{2} .
$$

\subsection{Anisotropy-with orthorhombic symmetry, $D_{2 h}$}

For the example of $\ell=3$, the matrix takes the form,

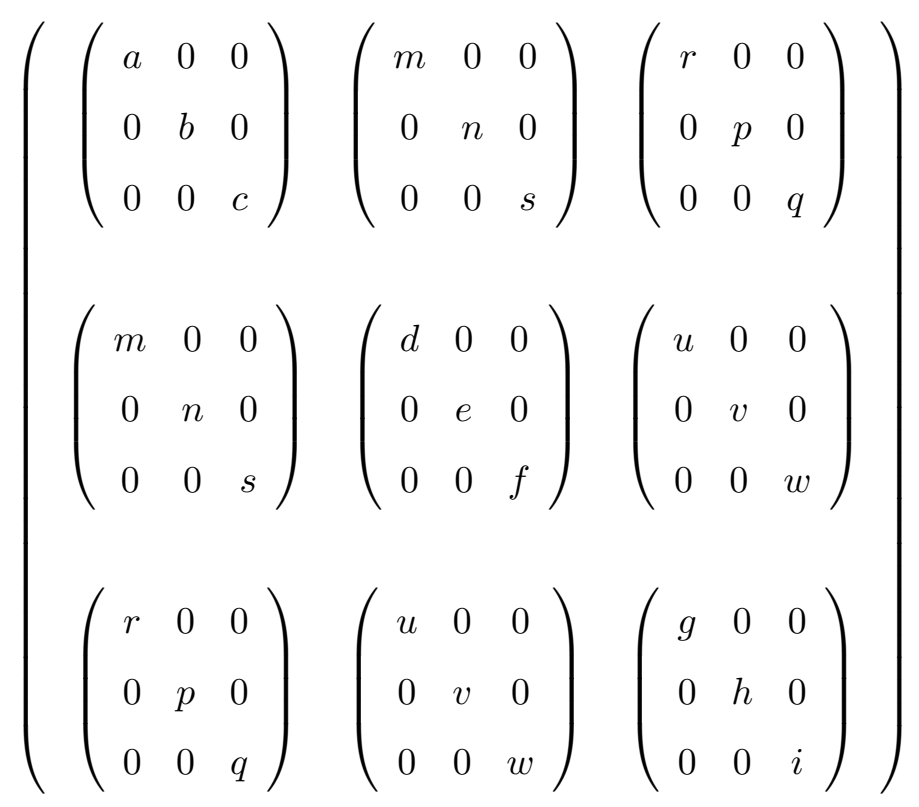

Here we have 18 independent coefficients, satisfying the 18 inequalities:

$$
a, b, c, d, e, f, g, h, i \geq 0
$$

$a d \geq m^{2}, \quad b e \geq n^{2}, \quad c f \geq s^{2}, \quad a g \geq r^{2}, \quad b h \geq p^{2}, \quad c i \geq q^{2}, \quad d g \geq u^{2}, \quad$ eh $\geq v^{2}, \quad f i \geq w^{2}$. 
For any $\ell$, the number of independent components is:

$$
\begin{array}{ccc}
\text { for symmetry group } & O(3): & \frac{1}{2} \ell(\ell+1) . \\
\text { for symmetry group } & D_{\infty h}: & \ell(\ell+1) . \\
\text { for symmetry group } & D_{2 h}: & \frac{3}{2} \ell(\ell+1) .
\end{array}
$$

It it is interesting to note that the material symmetries, $D_{4 h}$, and $D_{6 h}$, with horizontal honeycomb cross-section, which are close $D_{\infty h}$ have also $\ell(\ell+1)$ independent coefficients.

\section{Multiple fluid phases}

So far, the discussion has been related to phenomena of transport in saturated flow, i.e., in a fluid that fills up the entire pore space. However, often, a number of fluid phases occupy the void space simultaneously, each at at a certain saturation, where the saturation, $S_{\alpha}$ of an $\alpha$-phase is defined as the ratio between the volume of the $\alpha$-phase and the volume of the pore space, with $\sum_{\alpha} S_{\alpha}=1$. Under such conditions, considering phenomena of transport within a fluid phase, neglecting interphase transfers of the considered extensive quantities across (microscopic) interphase boundaries, each of the components: $K_{i j}, a_{i j k l}$, and all $L_{i j}^{q r}$ 's, is a function of the phase saturation, $S_{\alpha}$. The reason for this dependence stems from the fact that the geometric features of the phase occupied portion of the pore space, e.g. the tortuosity, varies with the saturation.

For example, for an anisotropic porous medium, with orthorhombic symmetry and with $x, y, z$ principal directions, $k_{x x}=f_{x}\left(S_{w}\right), k_{y y}=f_{y}\left(S_{w}\right), k_{z z}=f_{z}\left(S_{w}\right)$, but still, the $K_{x y}=f_{x y}\left(S_{w}\right)=$ $K_{y x}\left(S_{w}\right)$, etc. Another example is the dispersivity. $a_{i j k l}$. For an isotropic porous medium, we have $a_{L}=f_{a L}\left(S_{w}\right), a_{L}=f_{a T}\left(S_{w}\right)$, with $f_{a L} \neq f_{a T}$.

\section{Summary}

We have studied a number of transport coefficients that appear in the laws that govern advective, diffusive and dispersive fluxes of extensive quantities that are transported within a phase occupying the void space of a porous medium or part of it. We focused on an anisotropic porous medium. Making use of the basic features of tensors of 2nd and 4th rank, we have determined for each considered transport coefficient, the number of independent components. This information will enable further research on the relationships among the various dispersivity components. It will also facilitate the analysis of experiments that are conducted in order to determine the numerical values of these components by inverse methods.

In a separate article, Bear and Fel (2009) discuss the minimum number of experiments that is required in order to determine the values of the six $a_{i}$ moduli for the axially symmetric case. 


\section{Acknowledgement}

The authors wish to thank Prof. Alexander H.-D. Cheng, Department of Civil Engineering, University of Mississippi, Dr. Peter C. Lichtner, Los Alamos National Laboratory, and Prof. M. Poreh, Department of Civil and Environmental Engineering, Technion-Israel Institute of Technology, for fruitful discussions. The research was partly supported by the Kamea Fellowship program.

\section{References}

[1] Batchelor, G. K. The Theory of Axisymmetric Turbulence, J. Proceedings of the Royal Society of London. Series A, Mathematical and Physical Sciences, 186(1007): 480-502, 1946.

[2] Bear, J. On the tensor form of dispersion, J. Geophys. Res., 66:1185-1197, 1961.

[3] Bear, J. Dynamics of Fluids in Porous Media, American Elsevier, 764 pp., 1972 (also published by Dover Publications, 1988).

[4] Bear, J. and Bachmat, Y. Introduction to Modeling of Transport Phenomena in Porous Media, Kluwer Academic Publishers, 553pp., 1990.

[5] Bear, J. and L. Fel. Dispersion and Dispersivity Tensors in Saturated Porous Media with Uniaxial Symmetry, http://xxx.lanl.gov/abs/0904.3447, 2009.

[6] De Groot, S. Thermodynamics of Irreversible Processes, North-Holland Publ. Company, Amsterdam, 242pp., 1963.

[7] De Groot, S. R. and Mazur, P. Non-Equilibrium Thermodynamics, North-Holland Publ. Company, Amsterdam, 510pp., 1962.

[8] Fel, L. G. Piezoelectricity and Piezomagnetism: Duality in two-dimensional checkerboards, J. Math. Phys., 43(5):2606-2609, 2002.

[9] Poreh, M. The dispersivity tensor in isotropic and axisymmetric mediums, Jour. Geophys. Res., 76(16): 3909-3913, 1965.

[10] Robertson, H. P. The invariant theory of isotropic turbulence, Proc. Phil. Soc., 36:209-223, 1940.

[11] Sirotine, Y. and Chaskolskaya, M., Fondaments de la physique des crystaux, Edition Mir, Moscow, 680pp., (Russian Ed., 1975) 1984 